\title{
Die Werteerziehung im Lehrwerk Johannes Pauls II.
}

\section{Upbringing towards values in the teachings of John Paul II}

\begin{abstract}
An in-depth analysis of writings of John Paul II leads to a conclusion that the Pope cared for upbringing that would foster to values. He clearly indicated that the person is to be viewed as the foundation of the process of upbringing and also stressed the significance of upbringing towards life in the family, nation and Church. The author of this article accounts for and presents the problem of upbringing towards values, which is interpreted from the teachings of John Paul II. First, the author indicates the complementary character of education, religion and faith, then they are oriented towards the world of values and the Christian hierarchization of these values.
\end{abstract}

Keywords

Upbringing, education, values, religion, John Paul II.

Die vertiefte Analyse der Texte Johannes Pauls II. führt zu der Erkenntnis, dass er sich als Philosoph, Theologe, Priester, Bischof und schließlich als Papst um die Werteerziehung gesorgt hat, wobei er ausdrücklich darauf verweist, dass die Person auf spezifische Weise als Fundament der Werteerziehung zu betrachten sei, insbesondere wenn es um die Erziehung auf das Leben in der Familie, in der Nation und in der Kirche hin geht. Daher ist es nur zu berechtigt, im folgenden Text den Fragenkreis um die Werteerziehung aufzugreifen, wie 
er dem Lehrwerk Johannes Pauls II. zu entnehmen ist. Dazu wird zunächst die gegenseitige Durchdringung von Erziehung, Religion und Glauben erörtert, bevor in den folgenden Abschnitten aufgezeigt wird, welchen Beitrag diese leisten, um in die Welt der Werte einzuführen und diese Werte dann in eine Hierarchie einzuordnen, die der christlichen Auffassung entspricht.

\section{Die gegenseitige Durchdringung von Erziehung, Religion und Glauben}

Die Charakteristik der Religion, die auf den Begriffen sich bilden, sich binden und bekennen basiert, wirft die Frage nach der Verbindung von Erziehung, Religion und Glauben auf. Diese spezifische Verbindung erfordert Überlegungen und Bewertungen unterschiedlicher Aspekte, die im vorliegenden Kapitel dargelegt werden. Falls die notwendige Bedingung für ein erfülltes Leben des Menschen darin besteht, unaufhörlich nach dessen eigentlichen Sinn zu fragen, dann kann man die kühne These aufstellen, dass Erziehung nicht ohne Religion und Religion nicht ohne Erziehung existierten kann ${ }^{1}$. Dabei ist jedoch zu beachten, dass Bildung keinerlei religiöse Begründung benötigt, da sie die Religiosität bereits auf entscheidende Weise selbst beinhaltet. Die Verbindung zwischen Erziehung und Religion bedeutet für die Pädagogik keine Betonung der ethischen Dimension ${ }^{2}$ sondern betrifft eher den systematischen religiösen Aspekt jeglicher Art von Erziehung sowie den erzieherischen Aspekt jeglicher

${ }^{1}$ Hier ist unter anderem auf folgende Autoren zu verweisen: F. Schweitzer, J. Frass, K. E. Nipkow, N. Perquina, Z. Trentie, P. Braido, M. J. Langrweld, A. Petzelt, P. Malavasi.

${ }^{2}$ Dabei geht es um den Standpunkt, dass pädagogisches Einwirken umso erfolgreicher ist, je mehr begründete und erwartete Auswirkungen es im Zeitraum der Erziehung hervorruft. Dadurch finden erzieherische Einflussnahme und selbstständiges Handeln keinen Widerhall als Grundsatz für Selbsterziehung der Person als Subjekt. Die Erziehung wird zur Ausbildung und Steuerung der Zöglinge. Eine ähnliche, wenn auch nicht direkt ableitbare Auffassung lässt sich an der Basis der polnischen Pädagogik in den Ansichten von W. Okoń finden. Dagegen entfaltet Alfons Benning seine Überlegungen auf der Grundlage der Anschauungen von Heinrich Dopp-Vorwald. Dessen pädagogische Ethik stellt die Frage nach den grundlegenden Werten, die jedes erzieherische Einwirken leiten und ihm Struktur verleihen. Er geht von der Annahme aus, dass sich ethische Werte bereits in der Existenz wiederfinden und aus der Bestimmung des Menschen zu einem verantwortungsbewussten Leben hervorgehen. Folglich entsteht die Sittlichkeit aus der Situtation des Menschen, Entscheidungen treffen zu müssen. Vgl. A. Benning, Ethik der Erziehung, Zürich 1992, S. 29. 
Art von Religion ${ }^{3}$. Selbstverständlich sind im Rahmen von Erziehung und Bildung Werte wie etwa Pflichtbewusstsein, Freiheit und Wahrheit. Ohne diese verlieren Lehrer und Schüler ihre Subjektivität, werden lediglich zu Mitteln, aber nicht zum Ziel im Lehr- und Lernprozess. Bei der Erziehung geht es weniger darum, die Beziehung zu den tatsächlichen Wahrheiten zu verstehen, als vielmehr das „Ich” der Person in Ordnung zu bringen und die Ordnung dieses „Ichs” selbst zu durchdringen ${ }^{4}$.

Die Religion unternimmt den Versuch, die Wirklichkeit aus der Sicht des Nächsten zu behandeln, der vernunftbegabt auf das Ziel hinsteuert, das ihm vorbestimmt ist, und sie so mit der eigenen menschlichen Welt zu verbinden. Auf ähnliche Weise fasst dies Paul Tillich ${ }^{5}$ auf, indem er unterstreicht, dass die Religion die Basis des menschlichen Lebens ist. Im weitesten und tiefsten Sinn des Wortes ist sie das, was uns unmittelbar berührt. Die Verbindung zwischen Religion und Erziehung erscheint hier als zweifache Perspektive eines einzigen Prozesses. Vom praktischen Gesichtspunkt aus funktioniert sie wie

${ }^{3}$ Vgl. J. Stala, E. Osewska, Anders erziehen in Polen. Der Erziehungs- und Bildungsbegriff im Kontext eines sich ständig verändernden Europas des XXI. Jahrhunderts, Tarnów 2009; Religious Education / Catechesis in the Family. A European Perspective, Hrsg. E. Osewska, J. Stala, Warszawa 2010; Nauki o rodzinie w stużbie rodziny, Hrsg. J. Stala, Kraków 2014; E. Osewska, Education and Internet Challenges, in: Education and Creativity, Hrsg. E. Osewska, Warszawa 2014, S. 43-56; J. Stala, Towards Religious Education in the Family in the 21st Century, in: The Contemporary Family: Local and European Perspectives, Hrsg. E. Osewska, J. Stala, Kraków 2015, S. 309-318; E. Osewska, To Educate in a Diversified Europe, „The Person and the Challenges“ 1 (2011) Nr. 1, S. 71-88 ; E. Osewska, The Current Situation of Education and Continuing Professional Development of RE Teachers in Poland in the Context of the Social Challenges, "The Person and the Challenges" 2 (2012) Nr. 2, S. 123-131.

${ }^{4}$ Vgl. J. Stala, Der Mensch als Person: Die bestimmende Grundlage für Johannes Paul II. in seinem Bild von der Familie, „The Person and the Challenges” 2 (2012) Nr. 2, S. 41-59; J. Stala, Lehrpläne und Schulbücher für den Religionsunterricht an den Staatlichen Grundschulen in Polen, „Bogoslovni vestnik“ 70 (2010) Nr. 3, S. 405-414; W. Okła, Interakcyjne uwarunkowania poczucia sensu życia, in: Człowiek - wartości - sens. Studia z psychologii egzystencji, Hrsg. K. Popielski, Lublin 1996, S. 419-422; T. Styczeń, Usensownić swe działanie to dla człowieka być, in: Człowiek - pytanie otwarte, Hrsg. K. Popielski, Lublin 1987, S. 177-180; L. Witkowski, Edukacja wobec sporów o (po) nowoczesność, Warszawa 1998, S. 107-120; T. Hejnicka Bezwińska, O zmianach w edukacji. Konteksty, zagrożenia i możliwości, Bydgoszcz 2000, S. 137-163; L. Nowak, Jednostka a system społeczny, in: Podmiotowość: możliwość, rzeczywistość, konieczność, Hrsg. P. Buczkowski, R. Cichocki, Poznań 1989, S. 71-108.

${ }^{5}$ Vgl. P. Tillich, Liebe Macht Gerechtigkeit, Walter de Gruyter - Berlin - New York 1991; P. Tillich, Religiöse Reden, Walter de Gruyter - Berlin - New York 1987; P. Tillich, Rzeczywistość Chrystusa. Nowy Byt, Oleśnica 1996; J. A. Łata, Odpowiadająca teologia Paula Tillicha, Oleśnica 1995. 
eine untrennbare Gesamtheit. Lediglich in der Theorie besteht die Möglichkeit, beide getrennt voneinander zu behandeln. Religion und Erziehung sind auf strukturelle Weise miteinander verknüpft. Denn der Mensch bewertet und hinterfragt den Sinn der bestimmten Erscheinung und des Rangs, den er in seinem Leben spielt. Sinn bedeutet dabei die Beziehung zur Wirklichkeit, über sie zu reflektieren schafft bereits Sinnhaftigkeit ${ }^{6}$. In dieser Bedeutung korrelieren Sinn und Sinnhaftigkeit des Sinns, insbesondere im Hinblick auf die Entscheidung für Werte und Gewissen.

Die Religion braucht auch die Erziehung. Eine Anforderung der Religion selbst besteht im Lernen, wie man denkt, argumentiert und bewertet ${ }^{7}$. Das bedeutet ebenso, dass die Gewissensbildung ein Postulat der Religion darstellt. Die Religion soll nicht jeden Handlungsinhalt und -hinweis exakt festlegen. Denn in diesem Fall könnte das Handeln „aus dem Bauwerk der Religion” heraus bedeuten, dass man sich aus dem eigenständigen Denken und Bewerten entlässt, also das Subjekt von der eigenen Autorität verabschiedet und diese durch eine äußere (eben religiöse) Autorität zu ersetzt. Als Konsequenz daraus würde sich Erziehung lediglich ausschnitthaft, instrumentell oder funktionell realisieren. Darüber hinaus ist nicht zu vergessen, dass Religiosität für das Subjekt bedeutet, Verantwortung für sich selbst und für die Gestaltung seines Lebens zu übernehmen. Daher kann und soll der Mensch als Person den religiösen Aspekt seiner Bildung nicht bewusst übergehen. Bei der Religionserziehung muss das Knüpfen von Beziehungen zwischen den Personen einen bedeutenden Raum einnehmen, das was man als Praktizieren einer universellen Solidarität bezeichnen $\mathrm{kann}^{8}$.

\section{Erziehung und Bildung als Einführung in die Welt der Werte}

Der Mensch ist eine Person, die beständig alternativen Werten und Pseudowerten gegenübersteht. Darum ruft die Problematik der Werte breites Interesse hervor

${ }^{6}$ Vgl. K. Popielski, Człowiek: egzystencja podmiotowo-osobowa, in: Człowiek-wartościsens. Studia Z psychologii egzystencji, Hrsg. K. Popielski, Lublin 1996, S. 25-48.

${ }^{7}$ Vgl. M. Heitger, Braucht Bildung Religion - braucht Religion Bildung?, in: Bildung, Glaube und Wissen, Hrsg. G. Brinek, G. Schaufler, Innsbruck - Wien 1991, S. 108-109.

${ }^{8}$ Por. G. Milan, L'autorita e liberta nell educazione dell adolescente, „Nuova Umanita” (1989) Nr 64-65, S. 109-124; E Fromm, Zdrowe społeczeństwo, Warszawa 1996, S. 174-181. 
und das nicht nur unter Wissenschaftlern sondern auch unter gewöhnlichen Menschen, die in ihrem Leben vielen unterschiedlichen Möglichkeiten begegnen und häufig schwierige Entscheidungen treffen müssen. Es genügt bereits, die für unsere heutige Zeit so charakteristische „Vermischung der Werte” und eine Krise mancher Wertvorstellungen ebenso zu bemerken wie die fortwährende Suche nach neuartigen Erfahrungen und Werten. Obwohl über diese sogenannten „Werte” bis heute nicht allgemein gesprochen wurde, hatten Begriffe wie gut oder Gutes im Grunde der Sache stets ähnliche Geltungsbereiche. Die Frage nach den Werten ist also so alt wie die philosophische Reflexion. Es ließe sich nur schwerlich auf ein philosophisches System verweisen, das nicht auf irgendeine Art und Weise dieses Problem aufgegriffen hätte, indem es die eine oder andere Wertehierarchie eingeführt oder deren Fehlen angeprangert hätte 9

Der neuzeitliche Mensch, der nicht selten die Theologie vollständig negiert, sucht die Quelle des Wissens und der Werte bald in sich, bald in der empirischen Welt. Der Einfluss des Positivismus, der Fakten und Werte gegenüberstellt sowie objektives (empirisches) Wissen vom subjektiven Werteverständnis (Emotion) unterscheidet, hat wesentlich dazu beigetragen, dass diese neuartige Wertebetrachtung so populär werden konnte ${ }^{10}$.

Im Hinblick auf ihren großen praktischen Wert ist die Aufgabe einer Klassifikation der Werte jedoch keine rein akademische Übung. Der Preis dafür, dass diesem Problem nicht die nötige Aufmerksamkeit geschenkt wird, ist doch eine beträchtliche Konfusion innerhalb der gegenwärtigen axiologischen Abhandlungen ${ }^{11}$. Die aktuelle, häufig postmodernistische Moralphilosophie behandelt die gesamte Ethik global, da ihre Theoretiker nicht nur gegen ein konkretes Moralsystem auftreten sondern sich geradezu die komplette Zerstörung der Ethik als Ziel setzen ${ }^{12}$. Von einer hierarchischen Anordnung

9 Vgl. R. Darowski, Filozofia człowieka, Kraków 1992, S. 100; Mały słownik etyczny, Hrsg. S. Jedynak, Bydgoszcz 1994, S. 9; H. Borowski, Wartość jako przeżycie, Lublin 1992, S. 7.

${ }^{10}$ Vgl. L. Żuk-Łapinska, Problem konfliktu wartości, „Studia Filozoficzne” 6 (259) (1987), S. 73.

${ }^{11}$ Vgl. S. Marczuk, Problemy klasyfikacji wartości, „Studia Filozoficzne” 6 (259) (1987), S. 81 .

12 Vgl. Z. Sareło, Postmodernistyczny styl myślenia i życia, in: Postmodernizm. Wyzwanie dla chrześcijaństwa, Poznań 1995, S. 15. Es scheint, dass die ethischen Ansichten des Postmodernismus vor allem aus einer falsch verstandenen Anthropologie herrühren. Indem sie die Möglichkeit negiert, die Wahrheit kennenzulernen, nimmt diese Philosophie dem Menschen das, was in wesentlichem Ausmaß seine geistige Natur ausmacht. Vgl. B. Stanosz, 
guter Werte kann man hier nur im Hinblick auf die Kriterien neben den axiologischen sprechen, die mit gewissen speziellen psychischen, intellektuellen oder schließlich technischen Dispositionen des Individuums in Zusammenhang stehen. Man unternimmt lediglich den Versuch, die Relationen zwischen den Werten zu beschreiben ohne sie genau zu definieren. Der gesamte Problemkreis bezieht sich auf die Frage nach der Auswahl zwischen verschiedenen Zielen. Daraus ergibt sich, dass die Präferenzen intuitiv oder geradezu emotional festgelegt werden. Eine Sache wird erst dann zu einer guten, wenn sie von irgend Jemand subjektiv für gut befunden wird. Indem sie nur das als Wert anerkennen, was bestimmte Bedürfnisse des Menschen befriedigt, vergessen die Befürworter eines derartigen Standpunktes jedoch, dass die Wirklichkeit allein bereits Werte erfordert. Übrigens erfüllt die These über die konfliktbehaftete Natur der Werte, zusammen mit der Überzeugung, ihre Begründung sei unmöglich, eine „nützliche” Rolle. Denn dank ihrer Rätselhaftigkeit erleichtert sie es, vor schwierigeren Werten zu Gunsten leichterer ebenso zu resignieren wie vor gesellschaftlich anerkannten zu Gunsten individuell empfundener ${ }^{13}$. Bei der Erörterung der Werteproblematik darf man jedoch nicht übersehen, dass die Vision der modernen Welt, auch die der Wertevorstellungen, heute eine nahezu allmächtige Ökonomie des freien Marktes schafft ${ }^{14}$ und dass die Entchristianisierung ganzer Völker und Gesellschaften nicht nur den Verlust des Glaubens hervorruft sondern unvermeidlich moralisches Bewusstsein und sittliche Fähigkeiten verblassen lässt ${ }^{15}$.

Liberalna a chrześcijańska filozofia wartości, in: Z punktu widzenia humanizmu, Warszawa 1995, S. 105.

${ }^{13}$ Vgl. W. Stróżewski, Istnienie i wartość, Kraków 1981, S. 31; W. Stróżewski, W kreggu wartości, Kraków 1992, S. 109; L. Żuk-Łapinska, Problem konfliktu wartości, „Studia Filozoficzne” 6 (259) (1987), S. 75.

${ }^{14}$ Vgl. J. Kowalski, Etyka katolicka wobec neoliberalizmu, „Polonia Sacra” 5 (1999), S. 188-189. Es verändert sich gerade auf drastische Weise ebenso die Rolle, die die Religion in der heutigen Wirklichkeit spielt. Denn im Grunde der Sache hört sie bereits auf, die Basis für das sittliche Leben der Gesellschaften zu sein. Zu deren Merkmal werden dagegen immer deutlicher moralischer Permissivismus und Relativismus, in deren Licht zahlreiche Verhaltensmuster der Menschen für sittlich neutral und völlig frei von moralischer Bewertung gehalten werden. Vgl. J. Mariański, Kryzys moralny czy transformacja wartości. Studium socjologiczne, Lublin 2001, S. 176-177.

${ }^{15}$ Vgl. Veritatis Splendor 107. Der Bedarf nach jedweden ethischen Kodizes wird geradezu negiert, vielmehr unterstreicht man sogar die Schädlichkeit, moralische Grundsätze durch Dekret festzuschreiben. Sowohl auf der Ebene des Individuums als auch der Gesellschaft 
Das Christentum lenkt die Aufmerksamkeit heute wieder verstärkt darauf, dass das charakteristische Merkmal des Menschen gerade darin besteht, den engen aktuellen Horizont des Hier und Heute zu überschreiten. Diese einzigartige Inklination zur Transzendenz ist mit der Suche nach Werten verknüpft, die obwohl sie nicht in Kategorien zählbarer Vorteile aufzufassen sind - eben auf wesentliche Art und Weise über Reichtum und Gelingen des menschlichen Lebens entscheiden ${ }^{16}$.

Vor jeder Axiologie, die versucht Kriterien für eine mögliche Werteanordnung zu finden, stehen erhebliche Schwierigkeiten. Daher tritt immerzu das Problem auf, Momente zu entdecken, durch die sich eine spezifische Wertehierarchie leichter erstellen ließe. Der christliche Standpunkt - der realistische und der objektive - setzt voraus, dass Werte je nach ihrem Stellenwert in der Hierarchie und ihrer besonderen Attraktivität die Realisierung erfordern. In anderen Worten gesagt beinhalten sie einen derartigen Moment, der die Möglichkeit ausschließt, gegen sie zu handeln. Werte „rufen” beinahe nach einer Reaktion des Subjekts, nach einer spezifischen Haltung ihnen gegenüber ${ }^{17}$. Das Christentum will unablässig daran erinnern, dass der Mensch frei ist dank der Wahrheit ${ }^{18}$.

begegnen wir immer seltener absoluten sittlichen Normen sowie stabilen und kohärenten Wertesystemen.

16 Vgl. J. Życiński, Medytacje sokratejskie, Lublin 1991, S. 122.

17 Vgl. W. Stróżewski, Istnienie i wartość, Kraków 1981, S. 24-27; A. Szostek, Wokół godności, prawdy i miłości, Lublin 1998, S. 101.

18 Bei der Analyse der tatsächlichen Werte zeigt T. Styczeń aber gleichzeitig auf, dass universelle menschliche Werte immer auch christliche Werte sind. Er bemerkt weiter, dass derzeit jedoch Christentum und menschliche Werte einander gegenüber gestellt werden, was eine gewisse Furcht vor dem Adjektiv „christlich” im Zusammenhang mit der Sorge um die menschlichen Werte erzeugt. Dabei wird vergessen, dass der Christ ein Mensch ist, der daran glaubt, dass Gott, der Schöpfer des Menschen, selbst als Mensch - Jesus Christus unter uns gelebt hat. Dies hat er getan, um jedem Menschen das Geheimnis seiner Größe endgültig zu enthüllen, aber auch um ihm bis zum Grund hin das Ausmaß der Veruntreuung dieser Größe aufzuzeigen. Jesus Christus setzt voraus, dass der Mensch seine eigene Würde grundsätzlich kennt. Darum wird die Öffnung auf die höheren Werte hin und folglich auch die Bereitschaft dazu, diesen alles unterzuordnen, vom christlichen Gesichtspunkt aus zur Voraussetzung dafür, die eigene Menschlichkeit im Ganzen zu realisieren. Vgl. K. Wojtyła, Elementarz etyczny, Lublin 1983, S. 76; T. Styczeń, Człowiek i jego prawa w nauczaniu Jana Pawła II, czyli chrześcijanin wobec wartości ludzkich dziś (Część pierwsza), „Wychowawca” (1993) Nr. 2, S. 22-23. 


\section{Bildung und Erziehung bei der Anordnung der Wertehierarchie}

Ebenso kann man die aktuelle Theorie über den Menschen geradezu im Hinblick auf ihren schöpferischen Beitrag zur Wertefrage untersuchen. Der Mensch erschafft sich unablässig neu, indem er wie ein Künstler vorgeht, der auf die Partitur schaut und in der Melodie den darin niedergeschriebenen Klang zusammensetzt $^{19}$. Die Ethik ist eine Theorie der Werte, die einen besonderen Dialog zwischen dem Menschen als Wert an sich und der Welt der Werte führt, die außerhalb seiner selbst liegen ${ }^{20}$.

Damit die von der Person anerkannten Werte ihre entsprechende Rolle im Leben erfüllen können, ist es unerlässlich, diese hierarchisch anzuordnen. Durch die Rangordnung der Werte muss man den geistigen Werten vor den materiellen sowie den kulturellen Werten vor den ökonomischen Vorrang gewähren ${ }^{21}$. Dies erfordert folglich, zweckdienliche Bemühungen zu unternehmen, damit der Mensch die Wertehierarchie auch anerkennt und annimmt. Grundlage für eine so verstandene Wertehierarchie bilden die von der Person akzeptierten ethischen Ideale ${ }^{22}$. Diese Vorgehensweise soll von der Sorge begleitet werden, dass kein Widerspruch zwischen dem eigenem Wohl und dem Wohl der anderen entsteht. Dazu sollen auch die Möglichkeiten für die Weitergabe des Wertesystems mit den fundamentalen Bereichen der Sittlichkeitserziehung in Einklang stehen. Als Grundlage für die Sittlichkeitserziehung werden die vom Menschen anerkannten Werte ebenso betrachtet wie deren Anordnung nach einer bestimmten Hierarchie ${ }^{23}$. Selbst der Auswahl der Werte soll eine Einteilung nach ihrer Wichtigkeit vorangehen. In diesem Fall spricht man von einer anerkannten und angenommenen „Wertehierarchie”. Dadurch kann leichter unterschieden werden zwischen bedeutsameren Werten, die auch

19 Vgl. A. Bobko, Poszukiwanie prawdy o człowieku, „Znak” 3 (2001), S. 57.

20 Vgl. K. Rudzińska, Filozofia wartości J. Tischnera, „Studia filozoficzne” 4 (1988), S. 32.

21 Vgl. M. Śnieżyński, Wartości chrześcijańskie - wartości uniwersalne, „Wychowawca“ (1993) Nr. 6, S. 9.

22 Vgl. J. Mariański, Moralność w procesie przemian. Szkice socjologiczne, Warszawa 1990, S. 403.

23 Vgl. S. Kowalczyk, Teoria poznania, Sandomierz 1997, S. 34-35. 
höhere Werte genannt werden, und weniger bedeutsamen Werten, die man als niedere Werte bezeichnen $\mathrm{kann}^{24}$.

In diesem Kontext erscheint die Klassifizierung der Werte als interessant, die S. Witek vorstellt. Diese Klassifizierung basiert auf dem Herausheben der fundamentalsten Werte. Er nennt sie die physischen Werte, die es dem Menschen am besten ermöglichen, in der ihn umgebenden Welt grundsätzlich zu „funktionieren”. Der Autor teilt die physischen Werte in zwei Untergruppen ein: die Werte aus der biologischen und die aus der ökonomischen Sphäre des Menschen. Die zweite Gruppe der Werte verbindet S. Witek mit denjenigen Werten, die der weiteren Entwicklung der Person dienen. Er nennt sie die persönlichen Werte, um sie von den Werten abzugrenzen, die das reine Funktionieren des Menschen unterstützen. Hierbei unterscheidet er drei charakteristische Arten, die aus der Ebene der ästhetischen, der theoretischen und der technischen Dimension des menschlichen Lebens hervorgehen. Eine weitere Gruppe von Werten hängt nach S. Witek mit dem sozialen Leben des Menschen zusammen. Diese Gruppe untergliedert er in Werte hinsichtlich des politischen und des sozialen Lebens. Im Bezug zu diesen Lebensbereichen verweist er auf die Bedeutung von Werten wie etwa die öffentliche Ordnung, die Einhaltung von Recht und Gerechtigkeit sowie ein gut funktionierendes Gerichtswesen. $\mathrm{Zu}$ den sozialen Werten zählt er dagegen alle diejenigen, die dem Gemeinwohl dienen, insbesondere Freundschaft, Liebe und ein von Wohlwollen getragenes Zusammenleben der Menschen. Zur wichtigsten Wertegruppe gehören für S. Witek schließlich die Werte geistiger Natur. In wie weit der Mensch über sie verfügt, entscheidet über seine Reife und Vortrefflichkeit ${ }^{25}$. In der Gruppe der geistigen Werte listet der Autor Werte auf, die der ethischen sowie der religiösen Sphäre des menschlichen Lebens entspringen. Im Fall der erstgenannten geht es ihm um Werte in Verbindung mit dem Brauchtum und der Kultur, die den Menschen erst zu geeigneten Handlungsweisen befähigen. Dazu gehören vor allem Wahrheit und Freiheit. Die Sphäre der religiösen Werte drückt sich im Streben des Menschen nach persönlicher Heiligkeit aus, dessen Quelle das sacrum, Gott selbst ist.

\footnotetext{
${ }^{24}$ Vgl. A. Błasiak, Młodzież - świat wartości, Kraków 2002, S. 77-86.

${ }^{25}$ Vgl. K. Wojtyła, Elementarz etyczny, Lublin 1983, S. 73.
} 
Man kann das Thema der Werteerziehung, das im Lehrwerk des Papstes und damit des heiligen Johannes Pauls II. einen hohen Stellenwert einnimmt, nicht erörtern, ohne die grundlegenden Relationen zwischen Erziehung, Bildung, Axiologie und Religion zu betrachten. Darum wurde zuerst die gegenseitige Durchdringung von Erziehung, Religion und Glauben präsentiert, bevor im Folgenden auf die Einführung in die Welt der Werte durch Erziehung und Bildung sowie auf die hierarchische Anordnung der Werte eingegangen wurde.

\section{Bibliography}

Benning A., Ethik der Erziehung, Zürich 1992.

Błasiak A., Młodzież - świat wartości, Kraków 2002.

Bobko A., Poszukiwanie prawdy o człowieku, „Znak” 3 (2001), S. 57.

Borowski H., Wartość jako przeżycie, Lublin 1992.

Darowski R., Filozofia człowieka, Kraków 1992.

Education and Creativity, Hrsg. E. Osewska, Warszawa 2014.

Fromm E., Zdrowe społeczeństwo, Warszawa 1996.

Heitger M., Braucht Bildung Religion - braucht Religion Bildung?, in: Bildung, Glaube und Wissen, Hrsg. G. Brinek, G. Schaufler, Innsbruck - Wien 1991, S. 108-109.

Hejnicka - Bezwińska T., O zmianach w edukacji. Konteksty, zagrożenia i możliwości, Bydgoszcz 2000.

Kowalczyk S., Teoria poznania, Sandomierz 1997.

Kowalski J., Etyka katolicka wobec neoliberalizmu, „Polonia Sacra” 5 (1999), S. 188-189.

Łata J. A., Odpowiadająca teologia Paula Tillicha, Oleśnica 1995.

Mały słownik etyczny, Hrsg. S. Jedynak, Bydgoszcz 1994.

Marczuk S., Problemy klasyfikacji wartości, „Studia Filozoficzne” 6 (259) (1987).

Mariański J., Kryzys moralny czy transformacja wartości. Studium socjologiczne, Lublin 2001.

Mariański J., Moralność w procesie przemian. Szkice socjologiczne, Warszawa 1990.

Milan G., L'autorita e liberta nell educazione dell adolescente, „Nuova Umanita” (1989) Nr. 64-65, S. 109-124.

Nauki o rodzinie w stużbie rodziny, Hrsg. J. Stala, Kraków 2014.

Nowak L., Jednostka a system społeczny, in: Podmiotowość: możliwość, rzeczywistość, konieczność, Hrsg. P. Buczkowski, R. Cichocki, Poznań 1989, S. 71-108.

Osewska E., Education and Internet Challenges, in: Education and Creativity, Hrsg.

E. Osewska, Warszawa 2014, S. 43-56. 
Osewska E., The Current Situation of Education and Continuing Professional Development of RE Teachers in Poland in the Context of the Social Challenges, "The Person and the Challenges" 2 (2012) Nr. 2, S. 123-131.

Osewska E., To Educate in a Diversified Europe, „The Person and the Challenges“ 1 (2011) Nr. 1, S. 71-88.

Popielisk K., Człowiek: egzystencja podmiotowo-osobowa, in: Człowiek-wartości-sens. Studia Z psychologii egzystencji, Hrsg. K. Popielski, Lublin 1996, S. 25-48.

Religious Education / Catechesis in the Family. A European Perspective, Hrsg. E. Osewska, J. Stala, Warszawa 2010.

Rudzińska K., Filozofia wartości J. Tischnera, „Studia filozoficzne” 4 (1988), S. 32.

Sareło Z., Postmodernistyczny styl myślenia i życia, in: Postmodernizm. Wyzwanie dla chrześcijaństwa, Poznań 1995.

Stala J., Der Mensch als Person: Die bestimmende Grundlage für Johannes Paul II. in seinem Bild von der Familie, „The Person and the Challenges” 2 (2012) Nr. 2, S. 41-59.

Stala J., Lehrpläne und Schulbücher für den Religionsunterricht an den Staatlichen Grundschulen in Polen, „Bogoslovni vestnik“ 70 (2010) Nr. 3, S. 405-414.

Stala J., Osewska E., Anders erziehen in Polen. Der Erziehungs- und Bildungsbegriff im Kontext eines sich ständig verändernden Europas des XXI. Jahrhunderts, Tarnów 2009.

Stala J., Towards Religious Education in the Family in the 21st Century, in: The Contemporary Family: Local and European Perspectives, Hrsg. E. Osewska, J. Stala, Kraków 2015, S. 309-318.

Stanosz B., Liberalna a chrześcijańska filozofia wartości, in: Z punktu widzenia humanizmu, Warszawa 1995.

Stróżowski W., Istnienie i wartość, Kraków 1981.

Stróżowski W., W kręgu wartości, Kraków 1992.

Styczeń T., Człowiek i jego prawa w nauczaniu Jana Pawła II, czyli chrześcijanin wobec wartości ludzkich dziś (Część pierwsza), „Wychowawca” (1993) Nr. 2, S. 22-23.

Styczeń T., Usensownić swe działanie to dla człowieka być, in: Człowiek-pytanie otwarte, Hrsg. K. Popielski, Lublin 1987, S. 177-180.

Szostek A., Wokół godności, prawdy i miłości, Lublin 1998.

Śnieżyński M., Wartości chrześcijańskie - wartości uniwersalne, „Wychowawca” (1993) Nr. 6, S. 9.

Tillich P., Liebe Macht Gerechtigkeit, Walter de Gruyter - Berlin - New York 1991.

Tillich P., Religiöse Reden, Walter de Gruyter - Berlin - New York 1987.

Tillich P., Rzeczywistość Chrystusa. Nowy Byt, Oleśnica 1996.

Witkowski L., Edukacja wobec sporów o (po) nowoczesność, Warszawa 1998.

Wojtyła K., Elementarz etyczny, Lublin 1983.

Żuk-Łapinska L., Problem konfliktu wartości, „Studia Filozoficzne” 6 (259) (1987), S. 73.

Życiński J., Medytacje sokratejskie, Lublin 1991. 